Article

\title{
On Weak Limiting Distributions for Random Walks on a Spider
}

\author{
Youngsoo Seol \\ Department of Mathematics, Dong-A University, Busan 55037, Korea; prosul76@dau.ac.kr
}

Received: 8 October 2020; Accepted: 3 December 2020; Published: 4 December 2020

\begin{abstract}
In this article, we study random walks on a spider that can be established from the classical case of simple symmetric random walks. The primary purpose of this article is to establish a functional central limit theorem for random walks on a spider and to define Brownian spider as the resulting weak limit. In special case, random walks on a spider can be characterized as skew random walks. It is well known for skew Brownian motion as the resulting weak limit of skew random walks. We first will study the tightness and then it will be shown for the convergence of finite dimensional distribution for random walks on a spider.
\end{abstract}

Keywords: random walks on a spider; Brownian spider; skew random walks; skew Brownian motions; functional central limit theorem; tightness

\section{Introduction and the Statement of the Main Results}

Itô and Mckean [1] first proposed an elementary but interesting stochastic process which is named for Skew Brownian motion to provide a construction of some random processes related to Feller's classification of differential operators of the second order associated with diffusion processes (see Section 4.2 in [2]). In 1978, Walsh [3] expressed this model as a Brownian motion with excursions near zero in random directions which are values for random variables in $[0,2 \pi)$ and the different excursions with a constant price for each excursion are independent on the plane. Barlow, Pitman, and Yor [4] define more precisely the process which is called Walsh's Brownian motion.

From Evans and Sowers [5] and Barlow et al. [6], we propose a kind of Walsh's Brownian motion that occupies on $\mathrm{N}$ partial axes on the plane which is the so-called Walsh's spider, or Brownian spider. This motion behaves as a standard Brownian motion over each of the legs. Whenever the work arrives to the origin, with a given probability, it moves its motion over any of the $\mathrm{N}$ legs. Thus, someone can make the Brownian spider by independently setting the excursions from zero of a usual Brownian motion over the $\eta$-th leg of the spider with probability $p_{\eta}, \eta=1, \ldots, N$ with $\sum_{\eta=1}^{N} p_{\eta}=1$. For the special case of $p_{\eta}=1 / N, \eta=1, \ldots, N$, Papanicolaou et al. [7] established a general case of arc-sine law, regarding the time spent overall on the legs and observed the exit times for the specific sets. Vakeroudis and Yor [8] further developed this problem. Replacing the Brownian motions with the classical case of simple symmetric random walks over the legs, we realized that a counterpart of discrete cases for this motion is random walks on a spider. Hajri [9] investigated some versions for discrete case as asymptotic results of the Brownian spider and developed the weak limiting results in a more general case of asymptotic results for discrete cases that are related to Walsh's Brownian motion. He investigated that the results of weak limiting can be derived from skew random walks in the special case of $\mathrm{N}=2$ resulting from the convergence to a skew Brownian motion.

Matzavinos, Roitershtein, and Seol [10] first proposed random walks on a sparse random environment that can be perturbations of simple symmetric random walks and investigated a variety of asymptotic behaviors of this model, such as the existence of the asymptotic velocity, transience, and 
recurrence criteria and a transition of phase for their values, and limit theorems for some behaviors in both transient and recurrent regimes. Skew random walks specifically can be characterized as a special case of random walks in a fixed sparse environment with a certain deterministic sequence of constants.

In continuous cases, a relative similar process is the multi-dimensional skewed Brownian motion considered in Ramirez [11]. An analogue of direct discrete time for multi-dimensional skewed Brownian motion is a multi-dimensional skewed random walk, which can be expressed as a quenched type of the model with a certain deterministic expression of constants. In Ramirez [11], the marked sites are named interfaces whenever the long stretches of the usual sites divided by the interfaces are mentioned as layers that appeal to a motivation for physical meaning of the model.

Csáki et al. [12] proved an invariance principle with a strong version for the random walks on a spider by the Brownian spider as the limiting results. In addition, they investigated the transition probabilities and they also studied how high the walk can go over a spider with $\mathrm{N}$ legs, when $\mathrm{N}$ is fixed, and they also studied the probability that the walk goes up to certain heights concurrently over all legs when the number of legs are going to increase. In another Csáki et al. paper [13], they established limiting behaviors regarding occupation and local times on the legs when the number of steps go to infinity.

We start with a general description of random walks on a spider. Consider the following set of half lines, on the complex plane,

$$
\mathbb{S}_{N}:=\left\{v_{N}(x, \eta), x \geq 0, \eta=1,2, \ldots, N\right\},
$$

where, with $i=\sqrt{-1}$,

$$
v_{N}(x, \eta)=x\left(\exp \frac{2 \pi i(\eta-1)}{N}\right)
$$

We will call $\mathbb{S}_{N}$ a spider with $\mathrm{N}$ legs. In addition,

$$
v_{N}(0):=v_{N}(0,1)=v_{N}(0,2)=\cdots=v_{N}(0, N)
$$

is named the body of the spider, and $L_{\eta}:=\left\{v_{N}(x, \eta), x>0\right\}$ is the $\eta$-th leg of the spider. On $\mathbb{S}_{N}$, the distance can be defined as

$$
\left|v_{N}(x, \eta)-v_{N}(y, \eta)\right|=|x-y|, \eta=1 \ldots . ., N,
$$

and

$$
\left|v_{N}(x, \eta)-v_{N}(y, k)\right|=x+y, \quad \eta, k=1, \ldots N, \quad \eta \neq k .
$$

In this paper, $S_{n}^{\left(\alpha_{\eta}\right)}$ will stand for random walks on a spider and $S_{n}$ for a classical case of simple symmetric random walk on the line, with probabilities denoted by $P$ and $\mathbb{P}$, respectively. We will state the following notation which we will use throughout the paper, for $\eta=1, \ldots, N$,

$$
v_{N}(x, \eta): \Leftrightarrow x^{*} \text { and } v_{N}(-x, \eta): \Leftrightarrow-x^{*},
$$

which mean that the sign of minus is the exact opposite half line of the specific site. The number of legs $\mathrm{N}$ in this paper is fixed, so we will suppress $\mathrm{N}$ in the notation. That is, $v_{N}(0, \eta)=0^{*}=0$ and $v_{N}(m, \eta)=m^{*}$ for $m \neq 0$.

Throughout the paper, we consider a random walk on a spider $S_{n}^{\left(\alpha_{\eta}\right)}$ that starts from the body of the spider, i.e., $S_{0}^{\left(\alpha_{\eta}\right)}=0^{*}=0$, with the following probabilities of transition:

$$
P\left(S_{n+1}^{\left(\alpha_{\eta}\right)}=1^{*} \mid S_{n+1}^{\left(\alpha_{\eta}\right)}=0^{*}\right)=\alpha_{\eta}, \eta=1, \ldots, N,
$$


with

$$
\sum_{\eta=1}^{N} \alpha_{\eta}=1
$$

and, for $r=1,2, \ldots, \eta=1, \ldots, N$,

$$
P\left(S_{n+1}^{\left(\alpha_{\eta}\right)}=(r+1)^{*} \mid S_{n}^{\left(\alpha_{\eta}\right)}=r^{*}\right)=P\left(S_{n+1}^{\left(\alpha_{\eta}\right)}=(r-1)^{*} \mid S_{n}^{\left(\alpha_{\eta}\right)}=r^{*}\right)=\frac{1}{2} .
$$

Let $\gamma_{i}, i=1, \ldots$ be independent and identically distributed(i.i.d.) random variables with $P\left(\gamma_{i}=\eta\right)=\alpha_{\eta}, \eta=1,2, \ldots, N$, that are independent of the classical case of simple symmetric random walk $S_{n}$. As a consequence, we can redefine random walk on a spider as $S_{0}^{\left(\alpha_{\eta}\right)}=0$,

$$
S_{n}^{\left(\alpha_{\eta}\right)}:=\sum_{m=1}^{\infty} \mathbf{1}_{\left\{n \in G_{m}\right\}} v_{N}\left(\left|S_{n}\right|, \gamma_{m}\right), n=1,2, \ldots,
$$

if $S_{n} \neq 0$, and $S_{n}^{\left(\alpha_{\eta}\right)}=0^{*}=0$ if $S_{n}=0$. The limiting process for the random walks on a spider is named as Brownian motion on spider, or simply Brownian spider that is a kind of Walsh's Brownian motion (see Walsh [3], and Csáki et al. [12]). The construction of the Brownian spider is the following progresses. The standard Brownian motion $\{|B(t)|, t \geq 0\}$ has a number of countable excursions from zero, and denote by $J_{1}, J_{2}, \ldots$, a fixed enumeration of the intervals for its excursion away from zero. Then, for any $t>0$ for which $B(t) \neq 0$, we have that $t \in J_{m}$ for one of the values of $m=1,2, \ldots$ Let $k_{m}, m=1,2, \ldots$, be independent and identically distributed(i.i.d.) random variables, independent of standard Brownian motion $B(\cdot)$ with $P\left(k_{m}=\eta\right)=p_{\eta}, \eta=1,2, \ldots, N$.

By placing the excursion whose interval is $J_{m}$ to the $k_{m}$-th leg of the spider $S P(N)$, we are setting up the Brownian spider $B_{t}^{\left(\alpha_{\eta}\right)}, t \geq 0$, that can defined as the following:

$$
B_{t}^{\left(\alpha_{\eta}\right)}:=\sum_{m=1}^{\infty} \mathbf{1}_{\left\{n \in J_{m}\right\}} v_{N}\left(|B(t)|, k_{m}\right), n=1,2, \ldots,
$$

and $B_{t}^{\left(\alpha_{\eta}\right)}:=0^{*}=0$, if $B(t)=0$. As the special case of $\mathrm{N}=2$, they can be expressed as the general case of skewed random walk and skewed Brownian motion. In this case, we let $\alpha_{1}=\alpha, \alpha_{2}=1-\alpha$; then, the skew random walk $\left\{S_{n}^{\alpha}, n=0,1,2 \ldots\right\}$ with parameter $\alpha$ can be defined as a Markov chain on $\mathrm{Z}$ with probabilities of transition

$$
\begin{gathered}
P(0,1)=\alpha, \quad P(0,-1)=1-\alpha, \\
P(x, x+1)=P(x, x-1)=\frac{1}{2}, \quad x= \pm 1,2, \ldots \ldots
\end{gathered}
$$

In the case of definition in Equation (4), the model can be expressed as $S_{n}^{\alpha_{\eta}}=0$, if $S(n)=0$, and

$$
S_{n}^{\alpha_{\eta}}:=\sum_{m=1}^{\infty} \mathbf{1}_{\left\{n \in G_{m}\right\}} \delta_{m}\left|S_{n}\right|, \quad \text { if } S_{n} \neq 0, n=1,2, \ldots
$$

where $\delta_{m}, m=1,2, \ldots$ are independent and identically distributed(i.i.d.) random variables with $P\left(\delta_{1}=1\right)=\alpha=1-P\left(\delta_{1}=-1\right)$, and $G_{1}, G_{2}, \ldots$ are excursion intervals of $S_{n}$. The skew Brownian motion $B_{n}^{\alpha_{\eta}}$ with parameter $\alpha$ can be established as the following progresses. Let $B(\cdot)$ be a standard Brownian motion on the line, and let $J_{1}, J_{2}, \ldots$ be the intervals for excursion from zero 
in $|B(\cdot)|$. Let $\delta_{m}, m=1,2, \ldots$ are independent and identically distributed (i.i.d.) random variables, independent of $B(\cdot)$ with $P\left(\delta_{1}=1\right)=\alpha=1-P\left(\delta_{1}=-1\right)$. Then,

$$
\begin{gathered}
B_{n}^{\alpha_{\eta}}:=\sum_{m=1}^{\infty} \mathbf{1}_{\left\{t \in J_{m}\right\}} \delta_{m}|B(t)|, \quad \text { if } B(t) \neq 0, n=1,2, \ldots, \\
B_{n}^{\alpha_{\eta}}(t):=0, \quad B(t)=0 .
\end{gathered}
$$

Harrison and Shepp [14] mentioned without proof that the functional central limit theorem for skew Brownian motion as the limiting process of a properly generated skewed random walk on $\mathrm{Z}$. This result has considered as a foundation for numerical algorithms tracking moving particle in a highly heterogeneous porous media; (see, for examples [15-17]). In [16], they suggested that tightness can be proved based on the second moments, but this method can't be used even in the classical case of simple symmetric random walk. Due to the insufficiency of probabilistic independence of the increments, we use a fourth moment proof method for even more of a challenge. Although, in more general frameworks, proofs of Functional central limit theorems have subsequently been obtained by other methods (see, for instance [18] and Section 6.2 in [19]), a simple self-contained proof for tightness of skew random walk has not been available in the literature. Recently, Seol [20] investigated an elementary proof of the tightness by using the method rely on a fourth order moment.

In this paper, we consider the random walks in a spider which is the general case of skew random walks defined in Equation (4), and we will prove the following two main results. Let $C\left(\mathbb{R}_{+}, \mathbb{R}\right)$ be the space of continuous functions from $\mathbb{R}_{+}=[0, \infty)$ into $\mathbb{R}$, equipped with the topology of uniform convergence on compact sets. For $n \in \mathbb{N}$, let $X_{n}^{\left(\alpha_{\eta}\right)} \in C\left(\mathbb{R}_{+}, \mathbb{R}\right)$ denote the following linear interpolation of $S_{[n t]}^{\left(\alpha_{\eta}\right)}$ for random walks on a spider:

$$
X_{n}^{\left(\alpha_{\eta}\right)}(t)=\frac{1}{\sqrt{n}}\left(S_{[n t]}^{\left(\alpha_{\eta}\right)}+(n t-[n t]) \cdot S_{[n t]+1}^{\left(\alpha_{\eta}\right)}\right) .
$$

Throughout the paper, we denote $[x]$ the integer part of a real number $x$.

Theorem 1. For any $\alpha_{\eta} \in(0,1)$, there exists a constant $C>0$, such that the inequality

$$
E\left|X_{n}^{\left(\alpha_{\eta}\right)}(t)-X_{n}^{\left(\alpha_{\eta}\right)}(s)\right|^{4} \leq C|t-s|^{2},
$$

holds uniformly for all $t, s>0$, and $n \in \mathbb{N}$.

The results stated Theorem 1 implies (see, for example [21] (p. 98)):

Corollary 1. The family of processes $X_{n}^{\left(\alpha_{\eta}\right)}, n \in \mathbb{N}$, is tight in $C\left(\mathbb{R}_{+}, \mathbb{R}\right)$.

The following theorem states the convergence of finite dimensional distribution for random walks on a spider.

Theorem 2. Let $B$ be a standard Brownian motion starting at $x$ with probability density function $p(t ; x, y)=\frac{1}{\sqrt{2 \pi t}} \exp \left(-\frac{(y-x)^{2}}{2 t}\right)$ and $B^{\left(\alpha_{\eta}\right)}$ a Brownian spider and let $\tau_{0^{*}}^{x^{*}}=\inf \left\{k: S_{k}=0^{*}, S_{0}=x^{*}\right\}$, then

$$
\lim _{n \rightarrow \infty} E_{x^{*}}\left[e^{i \xi \frac{S^{\left(\alpha_{\eta}\right)}}{\sqrt[n]{n}}} g\left(\frac{S_{[n t]}^{\left(\alpha_{\eta}\right)}}{\sqrt{n}}\right)\right]=E_{x}\left[e^{i \tau B_{t}^{\left(\alpha_{\eta}\right)}} g\left(B_{t}^{\left(\alpha_{\eta}\right)}\right)\right]
$$


for bounded and continuous function $g$.

The structure of this paper is organized as follows. Some important results to prove the main results in Section 2. In Section 3, we discuss two conditions to prove the main results which are the tightness and convergence of finite dimensional distribution for random walks on spider. Finally, the proofs for the main theorems are contained in Section 3.

\section{Some Preliminary Results}

In this section, we provide some key lemmas which can be used in the proof of our main result. The $n$-step transition probabilities of random walks on a spider can be described as the following statement:

Proposition 1. For $m \in Z, k>0$

$$
P\left(S_{k}^{\left(\alpha_{\eta}\right)}=m^{*}\right)=\left\{\begin{array}{lll}
\alpha_{\eta} \cdot P\left(\left|S_{k}\right|=m^{*}\right) & \text { if } & m^{*}>0 \\
\left(1-\alpha_{\eta}\right) \cdot P\left(\left|S_{k}\right|=-m^{*}\right) & \text { if } & m^{*}<0 \\
P\left(\left|S_{k}^{\left(\alpha_{\eta}\right)}\right|=0^{*}\right)=P\left(\left|S_{k}\right|=0^{*}\right) & \text { if } & m^{*}=0
\end{array}\right.
$$

The following are immediate corollaries of these relationships.

Corollary 2. For $k>0$, it holds true:

(a) $P\left(S_{k}^{\left(\alpha_{\eta}\right)}>0^{*}\right)=\alpha_{\eta} P\left(\left|S_{k}\right| \neq 0^{*}\right)$;

(b) $P\left(S_{k}^{\left(\alpha_{\eta}\right)}<0^{*}\right)=\left(1-\alpha_{\eta}\right) P\left(\left|S_{k}\right| \neq 0^{*}\right)$.

Proof. Using Proposition 1, we obtain

$$
\begin{aligned}
P\left(S_{k}^{\left(\alpha_{\eta}\right)}>0^{*}\right) & =\sum_{m^{*}=1}^{\infty} P\left(S_{k}^{\left(\alpha_{\eta}\right)}=m^{*}\right)=\alpha_{\eta} \sum_{m^{*}=1}^{\infty} P\left(\left|S_{k}\right|=m^{*}\right) \\
& =\alpha_{\eta} P\left(\left|S_{k}\right|>0^{*}\right)=\alpha_{\eta}\left(1-P\left(\left|S_{k}\right|=0^{*}\right)\right)
\end{aligned}
$$

and

$$
\begin{aligned}
P\left(S_{k}^{\left(\alpha_{\eta}\right)}<0^{*}\right) & =\sum_{m^{*}=1}^{\infty} P\left(S_{k}^{\left(\alpha_{\eta}\right)}=-m^{*}\right)=\left(1-\alpha_{\eta}\right) \sum_{m^{*}=1}^{\infty} P\left(\left|S_{k}\right|=m^{*}\right) \\
& =\left(1-\alpha_{\eta}\right) P\left(\left|S_{k}\right|>0^{*}\right)=\left(1-\alpha_{\eta}\right)\left(1-P\left(\left|S_{k}\right|=0^{*}\right)\right) .
\end{aligned}
$$

Corollary 3. For $m \in Z, k>0$, it satisfies

$$
E S_{k}^{\left(\alpha_{\eta}\right)}= \begin{cases}\left(2 \alpha_{\eta}-1\right) E\left|S_{2 m-1}\right| & \text { if } \mathrm{k}=2 \mathrm{~m}, \mathrm{~m}=1,2, \ldots, \\
\left(2 \alpha_{\eta}-1\right)\left(\begin{array}{c}
2 m \\
m
\end{array}\right) 2^{-2 m}+\left(2 \alpha_{\eta}-1\right) E\left|S_{2 m}\right| & \text { if } \mathrm{k}=2 \mathrm{~m}+1, \mathrm{~m}=0,1, \ldots\end{cases}
$$

Proof. We first assume that $k=2 m, m \leq 1$. From Proposition 1, we obtain 


$$
\begin{aligned}
E S_{2 m}^{\left(\alpha_{\eta}\right)} & =\sum_{k=1}^{m} E\left(S_{2 m}^{\left(\alpha_{\eta}\right)} \mid S_{2 m-1}^{\left(\alpha_{\eta}\right)}=(2 k-1)^{*}\right) p\left(S_{2 m-1}^{\left(\alpha_{\eta}\right)}=(2 k-1)^{*}\right) \\
& +\sum_{k=1}^{m} E\left(S_{2 m}^{\left(\alpha_{\eta}\right)} \mid S_{2 m-1}^{\left(\alpha_{\eta}\right)}=-(2 k-1)^{*}\right) p\left(S_{2 m-1}^{\left(\alpha_{\eta}\right)}=-(2 k-1)^{*}\right) \\
& =\alpha_{\eta} \sum_{k=1}^{m}(2 k-1)^{*} p\left(\left|S_{2 m-1}\right|=(2 k-1)^{*}\right)+\left(1-\alpha_{\eta}\right) \sum_{k=1}^{m}\left(-(2 k-1)^{*}\right) p\left(\left|S_{2 m-1}\right|=(2 k-1)^{*}\right) \\
& =\left(2 \alpha_{\eta}-1\right) E\left|S_{2 m-1}\right|
\end{aligned}
$$

For $k=2 m+1, k \leq 0$,

$$
\begin{aligned}
E S_{2 m+1}^{\left(\alpha_{\eta}\right)} & =E\left(S_{2 m+1}^{\left(\alpha_{\eta}\right)} \mid S_{2 m}^{\left(\alpha_{\eta}\right)}=0^{*}\right) P\left(S_{2 m}=0^{*}\right)+\sum_{k=1}^{m} E\left(S_{2 m+1}^{\left(\alpha_{\eta}\right)} \mid S_{2 m}^{\left(\alpha_{\eta}\right)}=(2 k)^{*}\right) p\left(S_{2 m}^{\left(\alpha_{\eta}\right)}=(2 k)^{*}\right) \\
& +\sum_{k=1}^{m} E\left(S_{2 m+1}^{\left(\alpha_{\eta}\right)} \mid S_{2 m}^{\left(\alpha_{\eta}\right)}=-(2 k)^{*}\right) p\left(S_{2 m}^{\left(\alpha_{\eta}\right)}=-(2 k)^{*}\right) \\
& =\left(2 \alpha_{\eta}-1\right)\left(\begin{array}{c}
2 m \\
m
\end{array}\right) 2^{-2 m}+\alpha_{\eta} \sum_{k=1}^{m}\left((2 k)^{*}\right) p\left(\left|S_{2 m}\right|=(2 k)^{*}\right) \\
& +\left(1-\alpha_{\eta}\right) \sum_{k=1}^{m}\left(-(2 k)^{*}\right) p\left(\left|S_{2 m}\right|=(2 k)^{*}\right) \\
& =\left(2 \alpha_{\eta}-1\right)\left(\begin{array}{c}
2 m \\
m
\end{array}\right) 2^{-2 m}+\left(2 \alpha_{\eta}-1\right) E\left|S_{2 m}\right|
\end{aligned}
$$

Corollary 4. Let $S^{\left(\alpha_{\eta}\right)}$ be the random walks on a spider defined in Equation (4), then, for $i<j$,

$$
P\left(S_{2 j}^{\left(\alpha_{\eta}\right)}=0^{*} \mid S_{2 i+1}^{\left(\alpha_{\eta}\right)}=1^{*}\right)=P\left(S_{2 j}^{\left(\alpha_{\eta}\right)}=0^{*} \mid S_{2 i+1}^{\left(\alpha_{\eta}\right)}=-1^{*}\right) .
$$

Proof. The strong Markov property makes this obvious. Let $\tau_{0}=\inf \left\{k \geq 1: S_{2 k}^{\left(\alpha_{\eta}\right)}=0^{*}\right\}$. Since $P\left(S_{2 j}^{\left(\alpha_{\eta}\right)}=\right.$ $\left.0^{*} \mid S_{2 i+1}^{\left(\alpha_{\eta}\right)}=1^{*}\right)=P_{1}\left(S_{2 j-2 i-1}^{\left(\alpha_{\eta}\right)}=0^{*}\right)$, it suffices to show that $\forall m \in Z, P_{1}\left(S_{2 m+1}^{\left(\alpha_{\eta}\right)}=0^{*}\right)=P_{-1}\left(S_{2 m+1}^{\left(\alpha_{\eta}\right)}=0^{*}\right)$.

$$
\begin{aligned}
P_{1}\left(S_{2 m+1}^{\left(\alpha_{\eta}\right)}=0^{*}\right) & =P_{1}\left(S_{2 m+1}^{\left(\alpha_{\eta}\right)}=0^{*}, \tau_{0} \leq 2 m+1\right) \\
& =\sum_{i=1}^{2 m+1} P\left(S_{2 m+1}^{\left(\alpha_{\eta}\right)}=0^{*}, \tau_{0}=i\right)=\sum_{i=1}^{2 m+1} P_{1}\left(\tau_{0}=i\right) P_{0}\left(S_{2 m+1-i}^{\left(\alpha_{\eta}\right)}=0^{*}\right) \\
& =\sum_{i=1}^{2 m+1} P_{-1}\left(\tau_{0}=i\right) P_{0}\left(S_{2 m+1-i}^{\left(\alpha_{\eta}\right)}=0^{*}\right)=\sum_{i=1}^{2 m+1} P_{-1}\left(S_{2 m+1}^{\left(\alpha_{\eta}\right)}=0^{*}, \tau_{0}=i\right) \\
& =P_{-1}\left(S_{2 m+1}^{\left(\alpha_{\eta}\right)}=0^{*}, \tau_{0} \leq 2 m+1\right)=P_{-1}\left(S_{2 m+1}^{\left(\alpha_{\eta}\right)}=0^{*}\right) .
\end{aligned}
$$

Corollary 5. Let $S^{\left(\alpha_{\eta}\right)}$ be the random walks on a spider defined in Equation (4); then, for $j<k$,

$\sum_{y^{*} \neq 0}\left[P\left(S_{2 k}^{\left(\alpha_{\eta}\right)}=0^{*}, S_{2 j+1}^{\left(\alpha_{\eta}\right)}=(y+1)^{*}, S_{2 j}^{\left(\alpha_{\eta}\right)}=y^{*}\right)-P\left(S_{2 k}^{\left(\alpha_{\eta}\right)}=0^{*}, S_{2 j+1}^{\left(\alpha_{\eta}\right)}=(y-1)^{*}, S_{2 j}^{\left(\alpha_{\eta}\right)}=y^{*}\right)\right]=0$.

Proof. First, consider the above summation for $y \neq 0$ as the sum of two parts. 


$$
\begin{aligned}
& \sum_{y^{*}>0}\left[P\left(S_{2 k}^{\left(\alpha_{\eta}\right)}=0^{*} \mid S_{2 j+1}^{\left(\alpha_{\eta}\right)}=(y+1)^{*}\right) P\left(S_{2 j+1}^{\left(\alpha_{\eta}\right)}=(y+1)^{*} \mid S_{2 j}^{\left(\alpha_{\eta}\right)}=y\right) P\left(S_{2 j}^{\left(\alpha_{\eta}\right)}=y^{*}\right)\right. \\
& \left.-P\left(S_{2 k}^{\left(\alpha_{\eta}\right)}=0^{*} \mid S_{2 j+1}^{\left(\alpha_{\eta}\right)}=(y-1)^{*}\right) P\left(S_{2 j+1}^{\left(\alpha_{\eta}\right)}=(y-1)^{*} \mid S_{2 j}^{\left(\alpha_{\eta}\right)}=y^{*}\right) P\left(S_{2 j}^{\left(\alpha_{\eta}\right)}=y^{*}\right)\right]
\end{aligned}
$$

and

$$
\begin{aligned}
& \sum_{y^{*}<0}\left[P\left(S_{2 k}^{\left(\alpha_{\eta}\right)}=0^{*} \mid S_{2 j+1}^{\left(\alpha_{\eta}\right)}=(y+1)^{*}\right) P\left(S_{2 j+1}^{\left(\alpha_{\eta}\right)}=(y+1)^{*} \mid S_{2 j}^{\left(\alpha_{\eta}\right)}=y^{*}\right) P\left(S_{2 j}^{\left(\alpha_{\eta}\right)}=y^{*}\right)\right. \\
& \left.-P\left(S_{2 k}^{\left(\alpha_{\eta}\right)}=0^{*} \mid S_{2 j+1}^{\left(\alpha_{\eta}\right)}=(y-1)^{*}\right) P\left(S_{2 j+1}^{\left(\alpha_{\eta}\right)}=(y-1)^{*} \mid S_{2 j}^{\left(\alpha_{\eta}\right)}=y^{*}\right) P\left(S_{2 j}^{\left(\alpha_{\eta}\right)}=y^{*}\right)\right] .
\end{aligned}
$$

For the term in Equation (9) for summation of $y^{*}<0$, let $y^{*}=-y^{\prime},\left(y^{\prime}>0\right)$ for the case $y^{*}<0$ give the following

$$
\begin{aligned}
& \frac{1}{2} \sum_{y^{\prime}>0}\left[\left(1-\alpha_{\eta}\right) P\left(S_{2 k}^{\left(\alpha_{\eta}\right)}=0^{*} \mid S_{2 j+1}^{\alpha_{\eta}}=-y^{\prime}+1\right) P\left(\left|S_{2 j}\right|=y^{\prime}\right)\right. \\
& \left.\quad-\left(1-\alpha_{\eta}\right) P\left(S_{2 k}^{\left(\alpha_{\eta}\right)}=0^{*} \mid S_{2 j+1}^{\left(\alpha_{\eta}\right)}=-y^{\prime}-1\right) P\left(\left|S_{2 j}\right|=y^{\prime}\right)\right] .
\end{aligned}
$$

The term in Equation (8) is

$$
\begin{aligned}
& \frac{1}{2} \sum_{y^{*}>0}\left[P\left(S_{2 k}^{\left(\alpha_{\eta}\right)}=0^{*} \mid S_{2 j+1}^{\left(\alpha_{\eta}\right)}=(y+1)^{*}\right) P\left(S_{2 j+1}^{\left(\alpha_{\eta}\right)}=(y+1)^{*} \mid S_{2 j}^{\left(\alpha_{\eta}\right)}=y^{*}\right) P\left(S_{2 j}^{\left(\alpha_{\eta}\right)}=y^{*}\right)\right. \\
& \left.\quad-P\left(S_{2 k}^{\left(\alpha_{\eta}\right)}=0^{*} \mid S_{2 j+1}^{\left(\alpha_{\eta}\right)}=(y-1)^{*}\right) P\left(S_{2 j+1}^{\left(\alpha_{\eta}\right)}=(y-1)^{*} \mid S_{2 j}^{\left(\alpha_{\eta}\right)}=y^{*}\right) P\left(S_{2 j}^{\left(\alpha_{\eta}\right)}=y^{*}\right)\right]
\end{aligned}
$$

Using Proposition 1 and Equations (10) and (11), we conclude that

$\sum_{y^{*} \neq 0}\left[P\left(S_{2 k}^{\left(\alpha_{\eta}\right)}=0^{*}, S_{2 j+1}^{\left(\alpha_{\eta}\right)}=(y+1)^{*}, S_{2 j}^{\left(\alpha_{\eta}\right)}=y^{*}\right)-P\left(S_{2 k}^{\left(\alpha_{\eta}\right)}=0^{*}, S_{2 j+1}^{\left(\alpha_{\eta}\right)}=(y-1)^{*}, S_{2 j}^{\left(\alpha_{\eta}\right)}=y^{*}\right)\right]=0$.

The next few lemmas can be used to establish "tightness".

Lemma 1. With probability one,

$$
E\left(S_{2 j+1}^{\left(\alpha_{\eta}\right)}-S_{2 j}^{\left(\alpha_{\eta}\right)} \mid S_{2 j}^{\left(\alpha_{\eta}\right)}\right)=\left(2 \alpha_{\eta}-1\right) 1_{\left[S_{2 j}^{\left(\alpha_{\eta}\right)}=0^{*}\right]} .
$$

\section{Proof.}

$$
\begin{aligned}
E\left(S_{2 j+1}^{\left(\alpha_{\eta}\right)}-S_{2 j}^{\left(\alpha_{\eta}\right)} \mid S_{2 j}^{\left(\alpha_{\eta}\right)}\right) & =P\left(S_{2 j+1}^{\left(\alpha_{\eta}\right)}-S_{2 j}^{\left(\alpha_{\eta}\right)}=1 \mid S_{2 j}^{\left(\alpha_{\eta}\right)}\right)-P\left(S_{2 j+1}^{\left(\alpha_{\eta}\right)}-S_{2 j}^{\left(\alpha_{\eta}\right)}=-1 \mid S_{2 j}^{\left(\alpha_{\eta}\right)}\right) \\
& =\left(\frac{1}{2}\right) \mathbf{1}_{\left[S_{2 j}^{\left(\alpha_{\eta}\right)} \neq 0^{*}\right]}+\left(\alpha_{\eta}\right) \mathbf{1}_{\left[S_{2 j}^{\left(\alpha_{\eta}\right)}=0^{*}\right]}-\left(\frac{1}{2}\right) \mathbf{1}_{\left[S_{2 j}^{\left(\alpha_{\eta}\right)} \neq 0^{*}\right]}-\left(1-\alpha_{\eta}\right) \mathbf{1}_{\left[S_{2 j}^{\left(\alpha_{\eta}\right)}=0^{*}\right]} \\
& =\left(2 \alpha_{\eta}-1\right) \mathbf{1}_{\left[S_{2 j}^{\left(\alpha_{\eta}\right)}=0^{*}\right]} .
\end{aligned}
$$

Lemma 2. If we define a sequence $\{q(k)\}_{k \in \mathbb{Z}_{+}}$as follows:

$$
g(k)=\left\{\begin{array}{lll}
0 & \text { if } & k \in \mathbb{N} \text { is odd } \\
\left(\begin{array}{c}
2 i \\
i
\end{array}\right) 2^{-2 i} & \text { if } & k=2 i \in \mathbb{N} \text { is even. }
\end{array}\right.
$$


then, for $j \leq k$, we have

$$
E\left(\mathbf{1}_{\left[S_{2 k}^{\left(\alpha_{\eta}\right)}=0^{*}\right]}\left(S_{2 j+1}^{\left(\alpha_{\eta}\right)}-S_{2 j}^{\left(\alpha_{\eta}\right)}\right) \mid S_{2 j}^{\left(\alpha_{\eta}\right)}\right)=\left(2 \alpha_{\eta}-1\right) g(k-j) \mathbf{1}_{\left[S_{2 j}^{\left(\alpha_{\eta}\right)}=0^{*}\right]} .
$$

Proof. First, consider the equation as two parts:

$$
\begin{aligned}
E\left(\mathbf{1}_{\left[S_{2 k}^{\left(\alpha_{\eta}\right)}=0^{*}\right]}\left(S_{2 j+1}^{\left(\alpha_{\eta}\right)}-S_{2 j}^{\left(\alpha_{\eta}\right)}\right) \mid S_{2 j}^{\left(\alpha_{\eta}\right)}\right) & =P\left(S_{2 k}^{\left(\alpha_{\eta}\right)}=0^{*}, S_{2 j+1}^{\left(\alpha_{\eta}\right)}=S_{2 j}^{\left(\alpha_{\eta}\right)}+1^{*} \mid S_{2 j}^{\left(\alpha_{\eta}\right)}\right) \\
& -P\left(S_{2 k}^{\left(\alpha_{\eta}\right)}=0^{*}, S_{2 j+1}^{\left(\alpha_{\eta}\right)}=S_{2 j}^{\left(\alpha_{\eta}\right)}-1^{*} \mid S_{2 j}^{\left(\alpha_{\eta}\right)}\right) .
\end{aligned}
$$

From Corollaries 4 and 5, we have

$$
\begin{aligned}
E\left(\mathbf{1}_{\left[S_{2 k}^{\left(\alpha_{\eta}\right)}=0^{*}\right]}\left(S_{2 j+1}^{\left(\alpha_{\eta}\right)}-S_{2 j}^{\left(\alpha_{\eta}\right)}\right) \mid S_{2 j}^{\left(\alpha_{\eta}\right)}\right) & =P\left(S_{2 k}^{\left(\alpha_{\eta}\right)}=0^{*} \mid S_{2 j+1}^{\left(\alpha_{\eta}\right)}=1^{*}\right) P\left(S_{2 j+1}^{\left(\alpha_{\eta}\right)}=1^{*} \mid S_{2 j}^{\left(\alpha_{\eta}\right)}=0^{*}\right) \mathbf{1}_{\left[S_{2 j}^{\left(\alpha_{\eta}\right)}=0^{*}\right]} \\
& -P\left(S_{2 k}^{\left(\alpha_{\eta}\right)}=0^{*} \mid S_{2 j+1}^{\left(\alpha_{\eta}\right)}=-1^{*}\right) P\left(S_{2 j+1}^{\left(\alpha_{\eta}\right)}=-1^{*} \mid S_{2 j}^{\left(\alpha_{\eta}\right)}=0^{*}\right) \mathbf{1}_{\left[S_{2 j}^{\left(\alpha_{\eta}\right)}=0^{*}\right]} \\
& \pm P\left(S_{2 k}^{\left(\alpha_{\eta}\right)}=0^{*} \mid S_{2 j+1}^{\left(\alpha_{\eta}\right)}=S_{2 j}^{\left(\alpha_{\eta}\right)} \pm 1^{*}, S_{2 j}^{\left(\alpha_{\eta}\right)}\right) \\
& \cdot P\left(S_{2 j+1}^{\left(\alpha_{\eta}\right)}=S_{2 j}^{\left(\alpha_{\eta}\right)} \pm 1^{*} \mid S_{2 j}^{\left(\alpha_{\eta}\right)}\right) \mathbf{1}_{\left[S_{2 j}^{\left(\alpha_{\eta}\right)} \neq 0^{*}\right]} \\
& =P\left(S_{2 k}^{\left(\alpha_{\eta}\right)}=0^{*} \mid S_{2 j+1}^{\left(\alpha_{\eta}\right)}=1^{*}\right)\left(\alpha_{\eta}\right) \mathbf{1}_{\left[S_{2 j}^{\left(\alpha_{\eta}\right)}=0^{*}\right]} \\
& -P\left(S_{2 k}^{\left(\alpha_{\eta}\right)}=0^{*} \mid S_{2 j+1}^{\left(\alpha_{\eta}\right)}=-1^{*}\right)\left(1-\alpha_{\eta}\right) \mathbf{1}_{\left[S_{2 j}^{\left(\alpha_{\eta}\right)}=0^{*}\right]} \\
& =\left(2 \alpha_{\eta}-1\right) P\left(S_{2 k}^{\left(\alpha_{\eta}\right)}=0 \mid S_{2 j+1}^{\left(\alpha_{\eta}\right)}=1^{*}\right) \mathbf{1}_{\left[S_{2 j}^{\left(\alpha_{\eta}\right)}=0^{*}\right]} \\
& =\left(2 \alpha_{\eta}-1\right) g(k-j) \mathbf{1}_{\left[S_{2 j}^{\left(\alpha_{\eta}\right)}=0^{*}\right]} .
\end{aligned}
$$

Lemma 3. Define a sequence $\{q(k)\}_{k \in \mathbb{Z}_{+}}$as follows:

$$
g(k)=\left\{\begin{array}{lll}
0 & \text { if } & k \in \mathbb{N} \text { is odd } \\
\left(\begin{array}{c}
2 i \\
i
\end{array}\right) 2^{-2 i} & \text { if } & k=2 i \in \mathbb{N} \text { is even. }
\end{array}\right.
$$

For $j \leq k$,

$$
E\left(\mathbf{1}_{\left[S_{2 k}^{\left(\alpha_{\eta}\right)}=0^{*}\right]}\left(S_{2 j+1}^{\left(\alpha_{\eta}\right)}-S_{2 j}^{\left(\alpha_{\eta}\right)}\right)\right)=\left(2 \alpha_{\eta}-1\right) g(k-j) g(j) .
$$

Proof. First, we have the following equation from the definition of $S^{\left(\alpha_{\eta}\right)}$;

$$
\begin{aligned}
E\left(\mathbf{1}_{\left[S_{2 k}^{\left(\alpha_{\eta}\right)}=0^{*}\right]}\left(S_{2 j+1}^{\left(\alpha_{\eta}\right)}-S_{2 j}^{\left(\alpha_{\eta}\right)}\right)\right) & =P\left(S_{2 k}^{\left(\alpha_{\eta}\right)}=0^{*}, S_{2 j+1}^{\left(\alpha_{\eta}\right)}=S_{2 j}^{\left(\alpha_{\eta}\right)}+1^{*}\right) \\
& -P\left(S_{2 k}^{\left(\alpha_{\eta}\right)}=0^{*}, S_{2 j+1}^{\left(\alpha_{\eta}\right)}=S_{2 j}^{\left(\alpha_{\eta}\right)}-1^{*}\right) \\
& =\sum_{y=-2 j}^{2 j}\left[P\left(S_{2 k}^{\left(\alpha_{\eta}\right)}=0^{*}, S_{2 j+1}^{\left(\alpha_{\eta}\right)}=(y+1)^{*}, S_{2 j}^{\left(\alpha_{\eta}\right)}=y^{*}\right)\right. \\
& \left.-P\left(S_{2 k}^{\left(\alpha_{\eta}\right)}=0^{*}, S_{2 j+1}^{\left(\alpha_{\eta}\right)}=(y-1)^{*}, S_{2 j}^{\left(\alpha_{\eta}\right)}=y^{*}\right)\right] .
\end{aligned}
$$


Using Corollary 5, we have

$$
\begin{aligned}
E\left(\mathbf{1}_{\left[S_{2 k}^{\left(\alpha_{\eta}\right)}=0^{*}\right]}\left(S_{2 j+1}^{\left(\alpha_{\eta}\right)}-S_{2 j}^{\left(\alpha_{\eta}\right)}\right)\right) & =P\left(S_{2 k}^{\left(\alpha_{\eta}\right)}=0^{*}, S_{2 j+1}^{\left(\alpha_{\eta}\right)}=1^{*}, S_{2 j}^{\left(\alpha_{\eta}\right)}=0^{*}\right) \\
& -P\left(S_{2 k}^{\left(\alpha_{\eta}\right)}=0^{*}, S_{2 j+1}^{\left(\alpha_{\eta}\right)}=-1^{*}, S_{2 j}^{\left(\alpha_{\eta}\right)}=0^{*}\right) .
\end{aligned}
$$

It follows from Corollary 4 and Lemma 2 that

$$
\begin{aligned}
E\left(\mathbf{1}_{\left[S_{2 k}^{\left(\alpha_{\eta}\right)}=0^{*}\right]}\left(S_{2 j+1}^{\left(\alpha_{\eta}\right)}-S_{2 j}^{\left(\alpha_{\eta}\right)}\right)\right) & =P\left(S_{2 k}^{\left(\alpha_{\eta}\right)}=0^{*} \mid S_{2 j+1}^{\left(\alpha_{\eta}\right)}=1^{*}\right) P\left(S_{2 j+1}^{\left(\alpha_{\eta}\right)}=1^{*} \mid S_{2 j}^{\left(\alpha_{\eta}\right)}=0^{*}\right) P\left(S_{2 j}^{\left(\alpha_{\eta}\right)}=0^{*}\right) \\
& \left.-P\left(S_{2 k}^{\left(\alpha_{\eta}\right)}=0^{*}\right) \mid S_{2 j+1}^{\left(\alpha_{\eta}\right)}=-1^{*}\right) P\left(S_{2 j+1}^{\left(\alpha_{\eta}\right)}=-1^{*} \mid S_{2 j}=0^{*}\right) P\left(S_{2 j}^{\left(\alpha_{\eta}\right)}=0^{*}\right) \\
& =\left(2 \alpha_{\eta}-1\right) P\left(S_{2 k}^{\left(\alpha_{\eta}\right)}=0^{*} \mid S_{2 j+1}^{\left(\alpha_{\eta}\right)}=1^{*}\right) P\left(S_{2 j}^{\left(\alpha_{\eta}\right)}=0^{*}\right) \\
& =\left(2 \alpha_{\eta}-1\right) g(k-j) g(j) .
\end{aligned}
$$

Remark 1. To prove the weak convergence, we need to show the convergence of finite dimensional distributions and tightness properties. In the case of $\alpha_{\eta}=\frac{1}{2}$ of $S^{\left(\alpha_{\eta}\right)}$, one can easily show the tightness as a consequence of the simple random walk to Brownian motion because the simple random walk has independent and identically distributed increments (with finite second moments). (e.g., see [22]). However, for the case of random walks on a spider, the increments are not independent. Thus, we introduce a proposition derived from the Karamata Tauberian Theorem to show tightness. Let $\phi$ be a measure on $[0, \infty)$. We define the Laplace transform $\widehat{\phi}(\lambda)$ of $\phi$ to be the real-valued function for $\lambda \geq c$ by $\widehat{\phi}(\lambda):=\int_{0}^{\infty} e^{-\lambda x} \phi(d x), \lambda>c$ where $c=\inf \left\{\lambda: \int_{0}^{\infty} e^{-\lambda x} \phi(d x)<\infty\right\}$.

The following propositions can be found in [20,22].

Proposition 2. If $\phi$ and $\psi$ are measures on $[0, \infty)$ such that $\widehat{\phi}(\lambda)$ and $\widehat{\psi}(\lambda)$ both exist for $\lambda>0$, then the convolution $\gamma=\phi * \psi$ has the Laplace transform $\widehat{\gamma}(\lambda)=\widehat{\phi}(\lambda) \widehat{\psi}(\lambda)$ for all $\lambda>0$.

In general, for the $n$-fold convolution $f_{1} * f_{2} \cdots * f_{n}$ of $n$ integrable functions $f_{1}, f_{2}, \ldots, f_{n}$, we have $\left(f_{1} * \widehat{f_{2} \cdots} * f_{n}\right)=\widehat{f_{1}} \widehat{f}_{2} \ldots \widehat{f_{n}}$.

For the measures of discrete case, one has the following consequence of Karamata Tauberian Theorem represented in terms of generating function, i.e., with $t=e^{-\lambda}$, (e.g., see ([22] (p. 118)) as a special case of Karamata Tauberian Theorem.

Proposition 3. Let $\widetilde{\phi}(t)=\sum_{n=0}^{\infty} \phi_{n} t^{n}, 0 \leq t<1$, where $\left\{\phi_{n}\right\}_{n=0}^{\infty}$ is a sequence of non-negative numbers. For $L$ slowly varying at infinity and $0 \leq \theta<\infty$ one has

$$
\widehat{\phi}(t) \sim(1-t)^{-\theta} L\left(\frac{1}{1-t}\right) \text { as } t \uparrow \Longleftrightarrow \sum_{j=0}^{n} \phi_{j} \sim \frac{1}{\Gamma(\theta)} n^{\theta} L(n) \text { as } n \rightarrow \infty .
$$

We are in a position to get the following key proposition and the proof can be found in [20].

Proposition 4. If we define a sequence $\{q(k)\}_{k \in \mathbb{Z}_{+}}$as follows:

$$
g(k)= \begin{cases}0 & \text { if } k \in \mathbb{N} \text { is odd, } \\
\left(\begin{array}{c}
2 i \\
i
\end{array}\right) 2^{-2 i} & \text { if } k=2 i \in \mathbb{N} \text { is even, }\end{cases}
$$

then, $\{q(k)\}_{k \in \mathbb{Z}_{+}}$satisfies that

(a) If $\phi(j)=g * g(j)$, then $\sum_{j=0}^{m} \phi(j) \sim m$; 
(b) If $\psi(j)=g * g * g * g(j)$, then $\sum_{j=0}^{m} \psi(j) \sim m^{2}$.

Proof. First, we note that

$$
\begin{aligned}
& \sum_{1 \leq i_{1}<i_{2}<i_{3} \leq k-j} E\left(S_{i_{3}+1}^{\left(\alpha_{\eta}\right)}-S_{i_{3}}^{\left(\alpha_{\eta}\right)}\right)^{2}\left(S_{i_{2}+1}^{\left(\alpha_{\eta}\right)}-S_{i_{2}}^{\left(\alpha_{\eta}\right)}\right)\left(S_{i_{1}+1}^{\left(\alpha_{\eta}\right)}-S_{i_{1}}^{\left(\alpha_{\eta}\right)}\right) \\
& =\sum_{0 \leq i_{1}<i_{2}<i_{3} \leq\left[\frac{k-j}{2}\right]} E\left(S_{2 i_{3}+1}^{\left(\alpha_{\eta}\right)}-S_{2 i_{3}}^{\left(\alpha_{\eta}\right)}\right)^{2}\left(S_{2 i_{2}+1}^{\left(\alpha_{\eta}\right)}-S_{2 i_{2}}^{\left(\alpha_{\eta}\right)}\right)\left(S_{2 i_{1}+1}^{\left(\alpha_{\eta}\right)}-S_{2 i_{1}}^{\left(\alpha_{\eta}\right)}\right) .
\end{aligned}
$$

From the fact that $S_{2 i_{3}+1}^{\left(\alpha_{\eta}\right)}-S_{2 i_{3}}^{\left(\alpha_{\eta}\right)}= \pm 1$ with probability 1 and Lemma 3,

$$
\begin{aligned}
E\left(S_{2 i_{2}+1}^{\left(\alpha_{\eta}\right)}-S_{2 i_{2}}^{\left(\alpha_{\eta}\right)}\right)\left(S_{2 i_{1}+1}^{\left(\alpha_{\eta}\right)}-S_{2 i_{1}}^{\left(\alpha_{\eta}\right)}\right) & =E\left[\left(S_{2 i_{1}+1}^{\left(\alpha_{\eta}\right)}-S_{2 i_{1}}^{\left(\alpha_{\eta}\right)}\right) E\left(S_{2 i_{2}+1}^{\left(\alpha_{\eta}\right)}-S_{2 i_{2}}^{\left(\alpha_{\eta}\right)} \mid S_{2 i_{2}}^{\left(\alpha_{\eta}\right)}\right)\right] \\
& =E\left[\left(S_{2 i_{1}+1}^{\left(\alpha_{\eta}\right)}-S_{2 i_{1}}^{\left(\alpha_{\eta}\right)}\right)\left(2 \alpha_{\eta}-1\right) \mathbf{1}_{\left[S_{2 i_{2}}^{\left(\alpha_{\eta}\right)}=0^{*}\right]}\right] \\
& =\left(2 \alpha_{\eta}-1\right) E\left[\left(\mathbf{1}_{\left[S_{2 i_{2}}^{\left(\alpha_{\eta}\right)}=0^{*}\right]}\left(S_{2 i_{1}+1}^{\left(\alpha_{\eta}\right)}-S_{2 i_{1}}^{\left(\alpha_{\eta}\right)}\right)\right]\right. \\
& =\left(2 \alpha_{\eta}-1\right)^{2} g\left(i_{2}-i_{1}\right) g\left(i_{1}\right) .
\end{aligned}
$$

Thus, we have

$$
\begin{aligned}
\sum_{0 \leq i_{1}<i_{2}<i_{3} \leq\left[\frac{k-j}{2}\right]}\left(2 \alpha_{\eta}-1\right)^{2} g\left(i_{2}-i_{1}\right) g\left(i_{1}\right) & \leq\left(2 \alpha_{\eta}-1\right)^{2} \sum_{i_{3}=0}^{\left[\frac{k-j}{2}\right]} \sum_{i_{2}=0}^{i_{3}-1} \sum_{i_{1}=0}^{i_{2}-1} g\left(i_{2}-i_{1}\right) g\left(i_{1}\right) \\
& =\left(2 \alpha_{\eta}-1\right)^{2} \sum_{i_{3}=0}^{\left[\frac{k-j}{2}\right]} \sum_{i_{2}=0}^{i_{3}-1} g * g\left(i_{2}\right) \\
& \leq\left(2 \alpha_{\eta}-1\right)^{2} \sum_{i_{3}=0}^{\left[\frac{k-j}{2}\right]\left[\frac{k-j}{2}\right]} g * g\left(i_{2}\right) \\
& \leq C\left(2 \alpha_{\eta}-1\right)^{2}\left(\frac{k-j}{2}\right)\left(\frac{k-j}{2}\right)=C^{\prime}|k-j|^{2},
\end{aligned}
$$

where $C^{\prime}=C \frac{\left(2 \alpha_{\eta}-1\right)^{2}}{4}$.

Lemma 4. For integers $0<i_{1}<i_{2}<i_{3}$,

$$
\begin{gathered}
\sum_{1 \leq i_{1}<i_{2}<i_{3} \leq k-j} E\left(S_{i_{3}+1}^{\left(\alpha_{\eta}\right)}-S_{i_{3}}^{\left(\alpha_{\eta}\right)}\right)^{2}\left(S_{i_{2}+1}^{\left(\alpha_{\eta}\right)}-S_{i_{2}}^{\left(\alpha_{\eta}\right)}\right)\left(S_{i_{1}+1}^{\left(\alpha_{\eta}\right)}-S_{i_{1}}^{\left(\alpha_{\eta}\right)}\right) \\
\leq C|k-j|^{2} \text { for some } C>0 .
\end{gathered}
$$

Proof. Note first that

$$
\begin{aligned}
& \sum_{1 \leq i_{1}<i_{2}<i_{3} \leq k-j} E\left(S_{i_{3}+1}^{\left(\alpha_{\eta}\right)}-S_{i_{3}}^{\left(\alpha_{\eta}\right)}\right)^{2}\left(S_{i_{2}+1}^{\left(\alpha_{\eta}\right)}-S_{i_{2}}^{\left(\alpha_{\eta}\right)}\right)\left(S_{i_{1}+1}^{\left(\alpha_{\eta}\right)}-S_{i_{1}}^{\left(\alpha_{\eta}\right)}\right) \\
& =\sum_{0 \leq i_{1}<i_{2}<i_{3} \leq\left[\frac{k-j}{2}\right]} E\left(S_{2 i_{3}+1}^{\left(\alpha_{\eta}\right)}-S_{2 i_{3}}^{\left(\alpha_{\eta}\right)}\right)^{2}\left(S_{2 i_{2}+1}^{\left(\alpha_{\eta}\right)}-S_{2 i_{2}}^{\left(\alpha_{\eta}\right)}\right)\left(S_{2 i_{1}+1}^{\left(\alpha_{\eta}\right)}-S_{2 i_{1}}^{\left(\alpha_{\eta}\right)}\right) .
\end{aligned}
$$

From the fact that $S_{2 i_{3}+1}^{\left(\alpha_{\eta}\right)}-S_{2 i_{3}}^{\left(\alpha_{\eta}\right)}= \pm 1$ with probability 1 and Lemma 3, 


$$
\begin{aligned}
E\left(S_{2 i_{2}+1}^{\left(\alpha_{\eta}\right)}-S_{2 i_{2}}^{\left(\alpha_{\eta}\right)}\right)\left(S_{2 i_{1}+1}^{\left(\alpha_{\eta}\right)}-S_{2 i_{1}}^{\left(\alpha_{\eta}\right)}\right) & =E\left[\left(S_{2 i_{1}+1}^{\left(\alpha_{\eta}\right)}-S_{2 i_{1}}^{\left(\alpha_{\eta}\right)}\right) E\left(S_{2 i_{2}+1}^{\left(\alpha_{\eta}\right)}-S_{2 i_{2}}^{\left(\alpha_{\eta}\right)} \mid S_{2 i_{2}}^{\left(\alpha_{\eta}\right)}\right)\right] \\
& =E\left[\left(S_{2 i_{1}+1}^{\left(\alpha_{\eta}\right)}-S_{2 i_{1}}^{\left(\alpha_{\eta}\right)}\right)\left(2 \alpha_{\eta}-1\right) \mathbf{1}_{\left[S_{2 i_{2}}^{\left(\alpha_{\eta}\right)}=0^{*}\right]}\right] \\
& =\left(2 \alpha_{\eta}-1\right) E\left[\left(\mathbf{1}_{\left[S_{2 i_{2}}^{\left(\alpha_{\eta}\right)}=0^{*}\right]}\left(S_{2 i_{1}+1}^{\left(\alpha_{\eta}\right)}-S_{2 i_{1}}^{\left(\alpha_{\eta}\right)}\right)\right]\right. \\
& =\left(2 \alpha_{\eta}-1\right)^{2} g\left(i_{2}-i_{1}\right) g\left(i_{1}\right) .
\end{aligned}
$$

Thus, we have

$$
\begin{aligned}
\sum_{0 \leq i_{1}<i_{2}<i_{3} \leq\left[\frac{k-j}{2}\right]}\left(2 \alpha_{\eta}-1\right)^{2} g\left(i_{2}-i_{1}\right) g\left(i_{1}\right) & \leq\left(2 \alpha_{\eta}-1\right)^{2} \sum_{i_{3}=0}^{\left[\frac{k-j}{2}\right]} \sum_{i_{2}=0}^{i_{3}-1} \sum_{i_{1}=0}^{i_{2}-1} g\left(i_{2}-i_{1}\right) g\left(i_{1}\right) \\
& =\left(2 \alpha_{\eta}-1\right)^{2} \sum_{i_{3}=0}^{\left[\frac{k-j}{2}\right]} \sum_{i_{2}=0}^{i_{3}-1} g * g\left(i_{2}\right) \\
& \leq\left(2 \alpha_{\eta}-1\right)^{2} \sum_{i_{3}=0}^{\left[\frac{k-j}{2}\right]\left[\frac{k-j}{2}\right]} \sum_{i_{2}=0}^{2} g * g\left(i_{2}\right) \\
& \leq C\left(2 \alpha_{\eta}-1\right)^{2} \frac{k-j}{2} \frac{k-j}{2}=C^{\prime}|k-j|^{2}
\end{aligned}
$$

where $C^{\prime}=C \frac{\left(2 \alpha_{\eta}-1\right)^{2}}{4}$.

Lemma 5. For integers $0<i_{1}<i_{2}<i_{3}<i_{4}$,

$$
\begin{gathered}
\sum_{1 \leq i_{1}<i_{2}<i_{3}<i_{4} \leq k-j} E\left(S_{i_{4}+1}^{\left(\alpha_{\eta}\right)}-S_{i_{4}}^{\left(\alpha_{\eta}\right)}\right)\left(S_{i_{3}+1}^{\left(\alpha_{\eta}\right)}-S_{i_{3}}^{\left(\alpha_{\eta}\right)}\right)\left(S_{i_{2}+1}^{\left(\alpha_{\eta}\right)}-S_{i_{2}}^{\left(\alpha_{\eta}\right)}\right)\left(S_{i_{1}+1}^{\left(\alpha_{\eta}\right)}-S_{i_{1}}^{\left(\alpha_{\eta}\right)}\right) \\
\leq C|k-j|^{2} \text { for some } C>0 .
\end{gathered}
$$

Proof. Note first that

$$
\begin{gathered}
\sum_{1 \leq i_{1}<i_{2}<i_{3}<i_{4} \leq k-j} E\left(S_{i_{4}+1}^{\left(\alpha_{\eta}\right)}-S_{i_{4}}^{\left(\alpha_{\eta}\right)}\right)\left(S_{i_{3}+1}^{\left(\alpha_{\eta}\right)}-S_{i_{3}}^{\left(\alpha_{\eta}\right)}\right)\left(S_{i_{2}+1}^{\left(\alpha_{\eta}\right)}-S_{i_{2}}^{\left(\alpha_{\eta}\right)}\right)\left(S_{i_{1}+1}^{\left(\alpha_{\eta}\right)}-S_{i_{1}}^{\left(\alpha_{\eta}\right)}\right) \\
=\sum_{0 \leq i_{1}<i_{2}<i_{3}<i_{4} \leq\left[\frac{k-j}{2}\right]} E\left(S_{2 i_{4}+1}^{\left(\alpha_{\eta}\right)}-S_{2 i_{4}}^{\left(\alpha_{\eta}\right)}\right)\left(S_{2 i_{3}+1}^{\left(\alpha_{\eta}\right)}-S_{2 i_{3}}^{\left(\alpha_{\eta}\right)}\right)\left(S_{2 i_{2}+1}^{\left(\alpha_{\eta}\right)}-S_{2 i_{2}}^{\left(\alpha_{\eta}\right)}\right)\left(S_{2 i_{1}+1}^{\left(\alpha_{\eta}\right)}-S_{2 i_{1}}^{\left(\alpha_{\eta}\right)}\right) .
\end{gathered}
$$

First, consider only 


$$
\begin{aligned}
& E\left(S_{2 i_{4}+1}^{\left(\alpha_{\eta}\right)}-S_{2 i_{4}}^{\left(\alpha_{\eta}\right)}\right)\left(S_{2 i_{3}+1}^{\left(\alpha_{\eta}\right)}-S_{2 i_{3}}^{\left(\alpha_{\eta}\right)}\right)\left(S_{2 i_{2}+1}^{\left(\alpha_{\eta}\right)}-S_{2 i_{2}}^{\left(\alpha_{\eta}\right)}\right)\left(S_{2 i_{1}+1}^{\left(\alpha_{\eta}\right)}-S_{2 i_{1}}^{\left(\alpha_{\eta}\right)}\right) \\
& \quad=E\left[\left(S_{2 i_{\eta}+1}^{\left(\alpha_{\eta}\right)}-S_{2 i_{3}}^{\left(\alpha_{\eta}\right)}\right)\left(S_{2 i_{\eta}+1}^{\left(\alpha_{\eta}\right)}-S_{2 i_{2}}^{\left(\alpha_{\eta}\right)}\right)\left(S_{2 i_{1}+1}^{\left(\alpha_{\eta}\right)}-S_{\left.2 i_{1}\right)}^{\left(\alpha_{\eta}\right)}\right) E\left(S_{2 i_{4}+1}^{\left(\alpha_{\eta}\right)}-S_{2 i_{4}}^{\left(\alpha_{\eta}\right)} \mid S_{2 i_{4}}^{\left(\alpha_{\eta}\right)}\right)\right] \\
& \quad=E\left[\left(S_{2 i_{3}+1}^{\left(\alpha_{\eta}\right)}-S_{2 i_{3}}^{\left(\alpha_{\eta}\right)}\right)\left(S_{2 i_{2}+1}^{\left(\alpha_{\eta}\right)}-S_{2 i_{2}}^{\left(\alpha_{\eta}\right)}\right)\left(S_{2 i_{1}+1}^{\left(\alpha_{\eta}\right)}-S_{2 i_{1}}^{\left(\alpha_{\eta}\right)}\right)\left(2 \alpha_{\eta}-1\right) \mathbf{1}_{\left[S_{2 i_{4}}^{\left(\alpha_{\eta}\right)}=0^{*}\right]}\right] \\
&=\left(2 \alpha_{\eta}-1\right) E\left[\left(S_{2 i_{2}+1}^{\left(\alpha_{\eta}\right)}-S_{2 i_{2}}^{\left(\alpha_{\eta}\right)}\right)\left(S_{2 i_{1}+1}^{\left(\alpha_{\eta}\right)}-S_{2 i_{1}}^{\left(\alpha_{\eta}\right)}\right) E\left(\mathbf{1}_{\left[S_{2 i_{4}}^{\left(\alpha_{\eta}\right)}=0^{*}\right]}\left(S_{2_{3}+1}^{\left(\alpha_{\eta}\right)}-S_{2_{3}}^{\left(\alpha_{\eta}\right)}\right) \mid S_{23}^{\left(\alpha_{\eta}\right)}\right)\right] \\
&=\left(2 \alpha_{\eta}-1\right) E\left[\left(\left(S_{2 i_{2}+1}^{\left(\alpha_{\eta}\right)}-S_{2 i_{2}}^{\left(\alpha_{\eta}\right)}\right)\left(S_{2 i_{1}+1}^{\left(\alpha_{\eta}\right)}-S_{2 i_{1}}^{\left(\alpha_{\eta}\right)}\right)\left(2 \alpha_{\eta}-1\right) g\left(i_{4}-i_{3}\right) \mathbf{1}_{\left[S_{2 i_{3}}^{\left(\alpha_{\eta}\right)}=0^{*}\right]}\right)\right] \\
&=\left(2 \alpha_{\eta}-1\right)^{2} g\left(i_{4}-i_{3}\right) E\left[\left(S_{2 i_{1}+1}^{\left(\alpha_{\eta}\right)}-S_{2 i_{1}}^{\left(\alpha_{\eta}\right)}\right) E\left(\mathbf{1}_{\left[S_{2 i_{3}}^{\left(\alpha_{\eta}\right)}=0^{*}\right]}\left(S_{2 i_{2}+1}^{\left(\alpha_{\eta}\right)}-S_{2 i_{2}}^{\left(\alpha_{\eta}\right)}\right) \mid S_{2 i_{2}}^{\left(\alpha_{\eta}\right)}\right)\right] \\
&=\left(2 \alpha_{\eta}-1\right)^{2} g\left(i_{4}-i_{3}\right) E\left[\left(S_{2 i_{1}+1}^{\left(\alpha_{\eta}\right)}-S_{2 i_{1}}^{\left(\alpha_{\eta}\right)}\right)\left(2 \alpha_{\eta}-1\right) g\left(i_{3}-i_{2}\right) \mathbf{1}_{\left[S_{2 i_{2}}^{\left(\alpha_{\eta}\right)}=0^{*}\right]}\right] \\
&=\left(2 \alpha_{\eta}-1\right)^{3} g\left(i_{4}-i_{3}\right) g\left(i_{3}-i_{2}\right) E\left(\mathbf{1}_{\left[S_{2 i_{2}}^{\left(\alpha_{\eta}\right)}=0^{*}\right]}\left(S_{2 i_{1}+1}^{\left(\alpha_{\eta}\right)}-S_{2 i_{1}}^{\left(\alpha_{\eta}\right)}\right)\right) \\
&=\left(2 \alpha_{\eta}-1\right)^{3} g\left(i_{4}-i_{3}\right) g\left(i_{3}-i_{2}\right)\left(2 \alpha_{\eta}-1\right) g\left(i_{2}-i_{1}\right) g\left(i_{1}\right) \\
&=\left(2 \alpha_{\eta}-1\right)^{4} g\left(i_{4}-i_{3}\right) g\left(i_{3}-i_{2}\right) g\left(i_{2}-i_{1}\right) g\left(i_{1}\right) .
\end{aligned}
$$

Then, we have

$$
\begin{gathered}
\sum_{0 \leq i_{1}<i_{2}<i_{3}<i_{4} \leq\left[\frac{k-j}{2}\right]} E\left(S_{2 i_{4}+1}^{\left(\alpha_{\eta}\right)}-S_{2 i_{4}}^{\left(\alpha_{\eta}\right)}\right)\left(S_{2 i_{3}+1}^{\left(\alpha_{\eta}\right)}-S_{2 i_{3}}^{\left(\alpha_{\eta}\right)}\right)\left(S_{2 i_{2}+1}^{\left(\alpha_{\eta}\right)}-S_{2 i_{2}}^{\left(\alpha_{\eta}\right)}\right)\left(S_{2 i_{1}+1}^{\left(\alpha_{\eta}\right)}-S_{2 i_{1}}^{\left(\alpha_{\eta}\right)}\right) \\
=\left(2 \alpha_{\eta}-1\right)^{4} \sum_{0 \leq i_{1}<i_{2}<i_{3}<i_{4} \leq\left[\frac{k-j}{2}\right]} g\left(i_{4}-i_{3}\right) g\left(i_{3}-i_{2}\right) g\left(i_{2}-i_{1}\right) g\left(i_{1}\right) \\
\leq\left(2 \alpha_{\eta}-1\right)^{4} \sum_{i_{4}=0}^{\left[\frac{k-j}{2}\right]} \sum_{i_{3}=0}^{i_{4}-1} \sum_{i_{2}=0}^{i_{3}-1} \sum_{i_{1}=0}^{i_{2}-1} g\left(i_{4}-i_{3}\right) g\left(i_{3}-i_{2}\right) g\left(i_{2}-i_{1}\right) g\left(i_{1}\right) .
\end{gathered}
$$

Now, using the general case of the 4 -fold convolution, the last term in inequality expressed above can be written as

$$
\begin{aligned}
& \left(2 \alpha_{\eta}-1\right)^{4} \sum_{i_{4}=0}^{\left[\frac{k-j}{2}\right] \sum_{i_{3}=0}-1} \sum_{i_{2}=0}^{i_{3}-1} \sum_{i_{1}=0}^{i_{2}-1} g\left(i_{2}-i_{1}\right) g\left(i_{1}\right) g\left(i_{3}-i_{2}\right) g\left(i_{4}-i_{3}\right) \\
& =\left(2 \alpha_{\eta}-1\right)^{4} \sum_{i_{4}=0}^{\left[\frac{k-j}{2}\right]} \sum_{i_{3}=0}^{i_{4}-1} \sum_{i_{3}=0} g * g\left(i_{2}\right) g\left(i_{3}-i_{2}\right) g\left(i_{4}-i_{3}\right) \\
& =\left(2 \alpha_{\eta}-1\right)^{4} \sum_{i_{4}=0}^{\left[\frac{k-j}{2}\right]} \sum_{i_{3}=0}^{i_{4}-1} g * g * g\left(i_{3}\right) g\left(i_{4}-i_{3}\right) \\
& =\left(2 \alpha_{\eta}-1\right)^{4} \sum_{i_{4}=0}^{\left[\frac{k-j}{2}\right]} g * g * g * g\left(i_{4}\right) .
\end{aligned}
$$

Hence, using Lemma 4, we conclude that

$$
\left(2 \alpha_{\eta}-1\right)^{4} \sum_{i_{4}=0}^{\left[\frac{k-j}{2}\right]} g * g * g * g\left(i_{4}\right)=\left(2 \alpha_{\eta}-1\right)^{4} C\left[\frac{k-j}{2}\right]^{2}=C^{\prime}|k-j|^{2},
$$

where $C^{\prime}=\frac{\left(2 \alpha_{\eta}-1\right)^{4} C}{4}$. 


\section{Proof of Main Results}

In this section, we provide a complete proof of two conditions: tightness and convergence of finite dimensional distribution by using some auxiliary results introduced in the previous sections. First, we show tightness condition and then convergence of finite dimensional distribution.

\subsection{Proof of Theorem 1}

We are now in a position to complete the proof of our first main result.

Completion of the Proof of Theorem 1. First, consider the case where $s=\frac{j}{n}<\frac{k}{n}=t$ are grid points and $E\left|\frac{S_{[n t]}^{\left(\alpha_{\eta}\right)}}{\sqrt{n}}-\frac{S_{[n s]}^{\left(\alpha_{\eta}\right)}}{\sqrt{n}}\right|^{4}=\frac{1}{n^{2}} E\left|S_{k}^{\left(\alpha_{\eta}\right)}-S_{j}^{\left(\alpha_{\eta}\right)}\right|^{4}=\frac{1}{n^{2}} E\left|\sum_{i=j}^{k-1}\left(S_{i+1}^{\left(\alpha_{\eta}\right)}-S_{i}^{\left(\alpha_{\eta}\right)}\right)\right|^{4}$. Then, the last term above can be separated by four parts and using the previous propositions, we can conclude that

$$
E\left|\frac{S_{[n t]}^{\left(\alpha_{\eta}\right)}}{\sqrt{n}}-\frac{S_{[n s]}^{\left(\alpha_{\eta}\right)}}{\sqrt{n}}\right|^{4} \leq \frac{1}{n^{2}} \sum_{i=j}^{k-1} 1+\frac{1}{n^{2}}\left(\begin{array}{c}
k-j \\
2
\end{array}\right)\left(\begin{array}{c}
k-j \\
2
\end{array}\right)+\frac{1}{n^{2}} C_{1}|k-j|^{2}+\frac{1}{n^{2}} C_{2}|k-j|^{2} \leq C_{3}|t-s|^{2},
$$

for a large enough constant $C_{3}>0$.

To complete the proof of Theorem 1, it remains to observe that, for non-grid points $t$ and $s$, one can use an approximation by neighbor grid points. Actually, the argument for approximations given in [21] (pp. 100-101) for normal random walks addresses this verbatim.

\subsection{Proof of Theorem 2}

Remark 2. If $B=\left\{B_{t}: t \geq 0\right\}$ is a standard Brownian motion starting at $x$, then $B_{t}$ is a Gaussian with probability density function $p(t ; x, y)=\frac{1}{\sqrt{2 \pi t}} \exp \left(-\frac{(y-x)^{2}}{2 t}\right)$ and if $B^{\left(\alpha_{\eta}\right)}=\left\{B_{t}^{\left(\alpha_{\eta}\right)}: t \geq 0\right\}$ is a Brownian spider, then $B_{t}^{\left(\alpha_{\eta}\right)}$ is a Markov process with continuous sample paths whose the probabilities of transition $p^{\left(\alpha_{\eta}\right)}(t ; x, y)$ (see, for instance [12] ( $\left.\left.p .8\right)\right)$ is given by

$$
p^{\left(\alpha_{\eta}\right)}\left(t ; x^{*}, y^{*}\right)= \begin{cases}\frac{1}{\sqrt{2 \pi t}} e^{-\frac{(y-x)^{2}}{2 t}}+\frac{\left(2 \alpha_{\eta}-1\right)}{\sqrt{2 \pi t}} e^{-\frac{(y+x)^{2}}{2 t}} & \text { if } x \geq 0, y \geq 0, \\ \frac{1}{\sqrt{2 \pi t}} e^{-\frac{(y-x)^{2}}{2 t}}-\frac{\left(2 \alpha_{\eta}-1\right)}{\sqrt{2 \pi t}} e^{-\frac{(y+x)^{2}}{2 t}} & \text { if } x \leq 0, y \leq 0, \\ \frac{2 \alpha_{\eta}}{\sqrt{2 \pi t} e^{-\frac{(y-x)^{2}}{2 t}}} & \text { if } x \leq 0, y \geq 0, \\ \frac{2\left(1-\alpha_{\eta}\right)}{\sqrt{2 \pi t}} e^{-\frac{(y-x)^{2}}{2 t}} & \text { if } x \geq 0, y \leq 0 .\end{cases}
$$

and

$$
p^{\left(\alpha_{\eta}\right)}\left(t ; x^{*}, \hat{y}^{*}\right)=\frac{2 \alpha_{\hat{\eta}}}{\sqrt{2 \pi t}} e^{-\frac{(y+x)^{2}}{2 t}} \quad \text { if } \eta \neq \hat{\eta}
$$

where $v_{N}(y, \hat{\eta}): \Leftrightarrow \hat{y}^{*}$

Lemma 6. Let $B$ be a standard Brownian motion starting at $x$ and $B^{\left(\alpha_{\eta}\right)}$ a Brownian spider, then we have

$$
E e^{i \xi\left|B_{t}^{\left(\alpha_{\eta}\right)}\right|}=E e^{-i \xi\left|B_{t}\right|}+2 i \alpha_{\eta} E \sin \left(\xi\left|B_{t}\right|\right), \quad t \geq 0
$$

Proof. By the definition and transition probabilities of $B^{\left(\alpha_{\eta}\right)}$, we have 


$$
\begin{aligned}
E e^{i \xi\left|B_{t}^{\left(\alpha_{\eta}\right)}\right|} & =\int_{R} e^{i \xi|y|} p^{\left(\alpha_{\eta}\right)}\left(t ; 0^{*}, y^{*}\right) d y \\
& =\int_{-\infty}^{0} \frac{\left(2-2 \alpha_{\eta}\right)}{\sqrt{2 \pi t}} e^{-i \tilde{\zeta} y^{*}} e^{-\frac{y^{2}}{2 t}} d y+\int_{0}^{\infty} \frac{2 \alpha_{\eta}}{\sqrt{2 \pi t}} e^{i \xi y^{*}} e^{-\frac{y^{2}}{2 t}} d y
\end{aligned}
$$

and, furthermore, using the properties of $B^{\left(\alpha_{\eta}\right)}$, we have

$$
\begin{aligned}
E e^{i \xi\left|B_{t}^{\left(\alpha_{\eta}\right)}\right|} & =\int_{-\infty}^{0} \frac{\left(2-2 \alpha_{\eta}\right)}{\sqrt{2 \pi t}} e^{-i \xi y^{*}} e^{-\frac{y^{2}}{2 t}} d y+\int_{0}^{\infty} \frac{2 \alpha_{\eta}}{\sqrt{2 \pi t}} e^{i \xi y^{*}} e^{-\frac{y^{2}}{2 t}} d y \\
& =2 \int_{-\infty}^{0} \frac{1}{\sqrt{2 \pi t}} e^{-i \xi y} e^{-\frac{y^{2}}{2 t}} d y-2 \alpha_{\eta} \int_{-\infty}^{0} \frac{1}{\sqrt{2 \pi t}} e^{-i \xi y^{*}} e^{-\frac{y^{2}}{2 t}} d y+2 \alpha_{\eta} \int_{0}^{\infty} \frac{1}{\sqrt{2 \pi t}} e^{i \xi^{*} y^{*}} e^{-\frac{y^{2}}{2 t}} d y \\
& =\int_{R} \frac{1}{\sqrt{2 \pi t}} e^{-i \xi\left|y^{*}\right|} e^{-\frac{y^{2}}{2 t}} d y-\frac{2 \alpha_{\eta}}{2} \int_{R} \frac{1}{\sqrt{2 \pi t}} e^{-i \xi\left|y^{*}\right|} e^{-\frac{y^{2}}{2 t}} d y+\frac{2 \alpha_{\eta}}{2} \int_{R} \frac{1}{\sqrt{2 \pi t}} e^{i \xi\left|y^{*}\right|} e^{-\frac{y^{2}}{2 t}} d y \\
& =\int_{R} \frac{1}{\sqrt{2 \pi t}} e^{-i \xi\left|y^{*}\right|} e^{-\frac{y^{2}}{2 t}} d y+\alpha_{\eta} \int_{R} \frac{1}{\sqrt{2 \pi t}}\left(e^{i \xi\left|y^{*}\right|}-e^{-i \xi\left|y^{*}\right|}\right) e^{-\frac{y^{2}}{2 t}} d y \\
& =\int_{R} \frac{1}{\sqrt{2 \pi t}} e^{-i \xi\left|y^{*}\right|} e^{-\frac{y^{2}}{2 t}} d y+2 \alpha_{\eta} i \int_{R} \frac{1}{\sqrt{2 \pi t}} \sin \left(\xi\left|y^{*}\right|\right) e^{-\frac{y^{2}}{2 t}} d y \\
& =E e^{-i \xi\left|B_{t}\right|}+2 i \alpha_{\eta} E \sin \left(\xi\left|B_{t}\right|\right) .
\end{aligned}
$$

Remark 3. Let $\left\{X_{t}^{n}: t \geq 0\right\}, n=1,2, \ldots$, and $\left\{X_{t}: t \geq 0\right\}$ be stochastic processes whose sample paths belong to a metric space $S$ with metric $\rho$ and $\mathcal{B}$ the Borel sigma field for $S$.The distributions of $\left\{X_{t}^{n}: t \geq 0\right\}$ and $\left\{X_{t}: t \geq 0\right\}$ defined on a probability space $(\Omega, \mathcal{F}, P)$ are probability measures $Q_{n}$ and $Q$, respectively, for $B \in \mathcal{B}$

$$
\begin{aligned}
& Q(B)=P\left(\left\{\omega \in \Omega:\left\{X_{t}(\omega): t \geq 0\right\} \in B\right\}\right) \\
& Q_{n}(B)=P\left(\left\{\omega \in \Omega:\left\{X_{t}^{n}(\omega): t \geq 0\right\} \in B\right\}\right) .
\end{aligned}
$$

Convergence in distribution of $\left\{X_{t}^{n}: t \geq 0\right\}$ to $\left\{X_{t}: t \geq 0\right\}$ has been defined as the sequence of real-valued random variables $Y_{n}:=f\left(\left\{X_{t}^{n}: t \geq 0\right\}\right)$ converges in distribution to $Y:=f\left(\left\{X_{t}: t \geq 0\right\}\right)$ for each continuous function $f: S \rightarrow \mathbb{R}$. However, an equivalent condition is that, for each bounded and continuous real-valued function $f: S \rightarrow \mathbb{R}$, one has $\lim _{n \rightarrow \infty} E f\left(\left\{X_{t}^{n}: t \geq 0\right\}\right)=E f\left(\left\{X_{t}: t \geq 0\right\}\right)$. To show, for fixed $t>0, \frac{1}{\sqrt{n}} S_{[n t]}^{\left(\alpha_{\eta}\right)} \rightarrow B_{t}^{\left(\alpha_{\eta}\right)}$ as $n \rightarrow \infty$, it suffices to show that $E\left\{\exp \left(i \xi \frac{1}{\sqrt{n}} S_{[n t]}^{\left(\alpha_{\eta}\right)}\right)\right\} \rightarrow E\left\{\exp \left(i \xi B_{t}^{\left(\alpha_{\eta}\right)}\right)\right\}$ as $n \rightarrow \infty$ for all real $\xi$ and $t \geq 0$.

One dimensional distribution at single $t>0$ is shown in the following corollary.

Corollary 6. For fixed $t>0, \frac{1}{\sqrt{n}} S_{[n t]}^{\left(\alpha_{\eta}\right)} \rightarrow B_{t}^{\left(\alpha_{\eta}\right)}$ as $n \rightarrow \infty$.

Proof. It suffices to show that $E\left\{\exp \left(i \xi \frac{1}{\sqrt{n}} S_{[n t]}^{\left(\alpha_{\eta}\right)}\right)\right\} \rightarrow E\left\{\exp \left(i \xi B_{t}^{\left(\alpha_{\eta}\right)}\right)\right\}$ as $n \rightarrow \infty$ for all real $\xi$. 


$$
\begin{aligned}
E\left\{\exp \left(i \xi \frac{1}{\sqrt{n}} S_{[n t]}^{\left(\alpha_{\eta}\right)}\right)\right\} & =1 P\left(\frac{1}{\sqrt{n}} S_{[n t]}^{\left(\alpha_{\eta}\right)}=0^{*}\right)+\sum_{m^{*}>0} e^{\frac{i \xi m^{*}}{\sqrt{n}}} P\left(\frac{1}{\sqrt{n}} S_{[n t]}^{\left(\alpha_{\eta}\right)}=m^{*}\right) \\
& +\sum_{m^{*}<0} e^{\frac{i \xi m^{*}}{\sqrt{n}}} P\left(\frac{1}{\sqrt{n}} S_{[n t]}^{\left(\alpha_{\eta}\right)}=-m^{*}\right) \\
& =P\left(\frac{1}{\sqrt{n}} S_{[n t]}=0^{*}\right)+\alpha_{\eta} \sum_{m^{*}>0} e^{\frac{i \xi m^{*}}{\sqrt{n}}} P\left(\frac{1}{\sqrt{n}}\left|S_{[n t]}\right|=m^{*}\right) \\
& +\left(1-\alpha_{\eta}\right) \sum_{m^{*}<0} e^{\frac{i \xi m^{*}}{\sqrt{n}}} P\left(\frac{1}{\sqrt{n}}\left|S_{[n t]}\right|=-m^{*}\right) .
\end{aligned}
$$

In the above last term, letting $m^{\prime}=-m^{*}$, then $\left(1-\alpha_{\eta}\right) \sum_{m^{\prime}>0} e^{-\frac{i \tilde{\xi} m^{\prime}}{\sqrt{n}}} P\left(\frac{1}{\sqrt{n}}\left|S_{[n t]}\right|=m^{\prime}\right)$ and so, using Lemma 6, we have

$$
\begin{aligned}
E\left\{\exp \left(i \xi \frac{1}{\sqrt{n}} S_{[n t]}^{\left(\alpha_{\eta}\right)}\right)\right\} & =P\left(\frac{1}{\sqrt{n}}\left|S_{[n t]}\right|=0^{*}\right)+\sum_{m^{*}>0} e^{-\frac{i \xi m^{*}}{\sqrt{n}}} P\left(\frac{1}{\sqrt{n}}\left|S_{[n t]}\right|=m^{*}\right) \\
& +\alpha_{\eta} \sum_{m^{*}>0}\left(e^{\frac{i \xi m^{*}}{\sqrt{n}}}-e^{-\frac{i \xi m^{*}}{\sqrt{n}}}\right) P\left(\frac{1}{\sqrt{n}}\left|S_{[n t]}\right|=m^{*}\right)=P\left(\frac{1}{\sqrt{n}}\left|S_{[n t]}\right|=0^{*}\right) \\
& +\sum_{m^{*}>0} e^{-\frac{i \xi m^{*}}{\sqrt{n}}} P\left(\frac{1}{\sqrt{n}}\left|S_{[n t]}\right|=m^{*}\right)+2 i \alpha_{\eta} \sum_{m^{*}>0} \sin \left(\frac{\xi m^{*}}{\sqrt{n}}\right) P\left(\frac{1}{\sqrt{n}}\left|S_{[n t]}\right|=m^{*}\right) \\
& =E e^{-i \xi \frac{\left|S_{[n t]}\right|}{\sqrt{n}}}+2 i \alpha_{\eta} E \sin \left(\frac{\xi\left|S_{[n t]}\right|}{\sqrt{n}}\right) \\
& =E e^{-i \xi\left|B_{t}\right|}+2 i \alpha_{\eta} E \sin \left(\xi\left|B_{t}\right|\right)=E e^{i \xi\left|B_{t}^{\left(\alpha_{\eta}\right)}\right| .}
\end{aligned}
$$

Completion of the Proof of Theorem 2. For $x>0$, consider $E_{x}\left[e^{i \frac{i s_{k}^{\left(\alpha_{\eta}\right)}}{\sqrt{n}}} g\left(\frac{s_{k}^{\left(\alpha_{\eta}\right)}}{\sqrt{n}}\right)\right]$.

$$
\begin{aligned}
E_{x}\left[e^{i \xi \frac{s_{k}^{\left(\alpha_{\eta}\right)}}{\sqrt{n}}} g\left(\frac{S_{k}^{\left(\alpha_{\eta}\right)}}{\sqrt{n}}\right)\right] & =E_{x} e^{i \xi \frac{S_{k}}{\sqrt{n}}} g\left(\frac{S_{k}}{\sqrt{n}}\right) \mathbf{1}_{\left[\tau_{0^{*}}^{x^{*}}>k\right]}+\sum_{j=1}^{k} E_{x}\left[e^{i \xi \frac{\xi_{k}^{\left(\alpha_{\eta}\right)}}{\sqrt{n}}} g\left(\frac{S_{k}}{\sqrt{n}}\right) \mid \tau_{0^{*}}^{x^{*}}\right] P\left(\tau_{0^{*}}^{x^{*}}=j\right) \\
& =E_{x} e^{i \xi \frac{S_{k}}{\sqrt{n}}} g\left(\frac{S_{k}}{\sqrt{n}}\right) \mathbf{1}_{\left[\tau_{0^{*}}^{x^{*}}>k\right]}+\sum_{j=1}^{k} E_{0} e^{i \xi \frac{s_{k-j}^{\left(\alpha_{\eta}\right)}}{\sqrt{n}}} g\left(\frac{S_{k-j}}{\sqrt{n}}\right) P\left(\tau_{0^{*}}^{x^{*}}=j\right) \\
& =E_{x} e^{i \xi \frac{S_{k}}{\sqrt{n}}} g\left(\frac{S_{k}}{\sqrt{n}}\right) \mathbf{1}_{\left[\tau_{0^{*}}^{x^{*}}>k\right]}+\sum_{j=1}^{k}\left[\alpha_{\eta} E_{0} e^{i \xi \frac{\left|S_{k-j}\right|}{\sqrt{n}}} g\left(\frac{\left|S_{k-j}\right|}{\sqrt{n}}\right)\right. \\
& \left.+\left(1-\alpha_{\eta}\right) E_{0} e^{-i \xi \frac{\left|S_{k-j}\right|}{\sqrt{n}}} g\left(-\frac{\left|S_{k-j}\right|}{\sqrt{n}}\right)\right] P\left(\tau_{0^{*}}^{x^{*}}=j\right) \\
& =E_{x} e^{i \xi \frac{S_{k}}{\sqrt{n}}} g\left(\frac{S_{k}}{\sqrt{n}}\right) \mathbf{1}_{\left[\tau_{0^{*}}^{x^{*}}>k\right]}+\alpha_{\eta} E_{x} e^{i \xi \frac{\left|S_{k}\right|}{\sqrt{n}}} g\left(\frac{\left|S_{k}\right|}{\sqrt{n}}\right) \mathbf{1}_{\left[\tau_{0^{*}}^{x^{*}} \leq k\right]} \\
& +\left(1-\alpha_{\eta}\right) E_{x} e^{-i \xi \frac{\left|S_{k}\right|}{\sqrt{n}}} g\left(-\frac{\left|S_{k}\right|}{\sqrt{n}}\right) \mathbf{1}_{\left[\tau_{0^{*}}^{x^{*}} \leq k\right]} .
\end{aligned}
$$

Since $\frac{S_{[n t]}}{\sqrt{n}} \rightarrow B_{t}$, the above last terms converge to the following:

$$
E_{x} e^{i \xi B_{t}} g\left(B_{t}\right) \mathbf{1}_{\left[\tau_{0^{*}}^{* *}>t\right]}+\alpha_{\eta} E_{x} e^{i \xi\left|B_{t}\right|} g\left(\left|B_{t}\right|\right) \mathbf{1}_{\left[\tau_{0^{*}}^{x^{*}} \leq t\right]}+\left(1-\alpha_{\eta}\right) E_{x} e^{-i \tau\left|B_{t}\right|} g\left(-\left|B_{t}\right|\right) \mathbf{1}_{\left[\tau_{0^{*}}^{x^{*}} \leq t\right]} .
$$


Furthermore, the last term Equation (12) can be modified as

$$
\begin{aligned}
E_{x} e^{i \xi B_{t}} g\left(B_{t}\right) \mathbf{1}_{\left[\tau_{0^{*}}^{x^{*}}>t\right]} & +\alpha_{\eta} E_{x} e^{i \xi\left|B_{t}\right|} g\left(\left|B_{t}\right|\right) \mathbf{1}_{\left[\tau_{0^{*}}^{x^{*}} \leq t\right]}+\left(1-\alpha_{\eta}\right) E_{x} e^{-i \xi\left|B_{t}\right|} g\left(-\left|B_{t}\right|\right) \mathbf{1}_{\left[\tau_{0^{*}}^{x^{*}} \leq t\right]} \\
& =E_{x} e^{i \xi^{\xi} B_{t}} g\left(B_{t}\right) \mathbf{1}_{\left[\tau_{0^{*}}^{x^{*}}>t\right]}+\alpha_{\eta} E_{x} e^{i \xi\left|B_{t}\right|} g\left(\left|B_{t}\right|\right)-\alpha_{\eta} E_{x} e^{i \zeta_{t} B_{t}} g\left(B_{t}\right) \mathbf{1}_{\left[\tau_{0^{*}}^{x^{*}}>t\right]} \\
& +\left(1-\alpha_{\eta}\right) E_{x} e^{-i \xi\left|B_{t}\right|} g\left(-\left|B_{t}\right|\right)-\left(1-\alpha_{\eta}\right) E_{x} e^{-i \xi B_{t}} g\left(-B_{t}\right) \mathbf{1}_{\left[\tau_{0}^{x}>t\right]} .
\end{aligned}
$$

Finally, Equation (12) has the following:

$$
\begin{aligned}
& E_{x} e^{i \xi^{\xi} B_{t}} g\left(B_{t}\right) \mathbf{1}_{\left[\tau_{0^{*}}^{x^{*}}>t\right]}+\alpha_{\eta} E_{x} e^{i \xi\left|B_{t}\right|} g\left(\left|B_{t}\right|\right) \mathbf{1}_{\left[\tau_{0^{*}}^{x^{*}} \leq t\right]}+\left(1-\alpha_{\eta}\right) E_{x} e^{-i \xi\left|B_{t}\right|} g\left(-\left|B_{t}\right|\right) \mathbf{1}_{\left[\tau_{0^{*}}^{x^{*}} \leq t\right]} \\
& =\left(1-\alpha_{\eta}\right) E_{x} e^{i \xi B_{t}} g\left(B_{t}\right) \mathbf{1}_{\left[\tau_{0^{*}}^{\tau^{*}}>t\right]}+\alpha_{\eta} E_{x} e^{i \xi\left|B_{t}\right|} g\left(\left|B_{t}\right|\right) \\
& +\left(1-\alpha_{\eta}\right) E_{x} e^{-i \xi\left|B_{t}\right|} g\left(-\left|B_{t}\right|\right)-\left(1-\alpha_{\eta}\right) E_{x} e^{-i \xi B_{t}} g\left(-B_{t}\right) \mathbf{1}_{\left[\tau_{0^{*}}^{x^{*}}>t\right]} \\
& =\left(1-\alpha_{\eta}\right)\left[\int_{0}^{\infty} e^{i \xi y^{*}} g\left(y^{*}\right) p\left(t ; x^{*}, y^{*}\right) d y-\int_{0}^{\infty} e^{i \xi y^{*}} g\left(y^{*}\right) p\left(t ; x^{*},-y^{*}\right) d y\right] \\
& +\alpha_{\eta}\left[\int_{0}^{\infty} e^{i \xi y^{*}} g\left(y^{*}\right) p\left(t ; x^{*}, y^{*}\right) d y+\int_{0}^{\infty} e^{i \tilde{\xi} y^{*}} g\left(y^{*}\right) p\left(t ; x^{*},-y^{*}\right) d y\right] \\
& +\left(1-\alpha_{\eta}\right)\left[\int_{0}^{\infty} e^{-i \xi y^{*}} g\left(-y^{*}\right) p\left(t ; x^{*}, y^{*}\right) d y+\int_{0}^{\infty} e^{-i \xi y^{*}} g\left(-y^{*}\right) p\left(t ; x^{*},-y^{*}\right) d y\right] \\
& -\left(1-\alpha_{\eta}\right)\left[\int_{0}^{\infty} e^{-i \xi y^{*}} g\left(-y^{*}\right) p\left(t ; x^{*}, y^{*}\right) d y-\int_{0}^{\infty} e^{-i \xi^{*} y^{*}} g\left(-y^{*}\right) p\left(t ; x^{*},-y^{*}\right) d y\right] \\
& =\int_{0}^{\infty} e^{i \zeta^{*}} g\left(y^{*}\right) p\left(t ; x^{*}, y^{*}\right) d y+\left(2 \alpha_{\eta}-1\right) \int_{0}^{\infty} e^{i \tilde{y}^{*}} g\left(y^{*}\right) p\left(t ; x^{*},-y^{*}\right) d y \\
& +2\left(1-\alpha_{\eta}\right) \int_{0}^{\infty} e^{-i \xi y^{*}} g\left(-y^{*}\right) p\left(t ; x^{*},-y^{*}\right) d y \text {. }
\end{aligned}
$$

Using the transition probabilities of Browian spider (see, for instance, [12] (p. 8)), we have

$$
\begin{aligned}
E_{x} e^{i \xi B_{t}^{\left(\alpha_{\eta}\right)}} g\left(B_{t}^{\left(\alpha_{\eta}\right)}\right) & =\int_{0}^{\infty} e^{i \tilde{\zeta} y^{*}} g\left(y^{*}\right) p\left(t ; x^{*}, y^{*}\right) d y+\left(2 \alpha_{\eta}-1\right) \int_{0}^{\infty} e^{i \tau y^{*}} g\left(y^{*}\right) p\left(t ; x^{*},-y^{*}\right) d y \\
& +2\left(1-\alpha_{\eta}\right) \int_{-\infty}^{0} e^{i \tau y^{*}} g\left(-y^{*}\right) p\left(t ; x^{*}, y^{*}\right) d y=\int_{0}^{\infty} e^{i \xi y^{*}} g\left(y^{*}\right) p\left(t ; x^{*}, y^{*}\right) d y \\
& +\left(2 \alpha_{\eta}-1\right) \int_{0}^{\infty} e^{i \xi y^{*}} g\left(y^{*}\right) p\left(t ; x^{*},-y^{*}\right) d y+2\left(1-\alpha_{\eta}\right) \int_{0}^{\infty} e^{-i \xi y^{*}} g\left(-y^{*}\right) p\left(t ; x^{*},-y^{*}\right) d y
\end{aligned}
$$

for $x>0$. Therefore, we conclude that

$$
\lim _{n \rightarrow \infty} E_{x}\left[e^{i \xi^{S^{\left(\alpha_{\eta}\right)}} \frac{[n t]}{\sqrt{n}}} g\left(\frac{S_{[n t]}^{\left(\alpha_{\eta}\right)}}{\sqrt{n}}\right)\right]=E_{x} e^{i \xi^{\left(\tau_{t}^{\left(\alpha_{\eta}\right)}\right.}} g\left(B_{t}^{\left(\alpha_{\eta}\right)}\right)
$$

for bounded and continuous function $\mathrm{g}$. Take $g \equiv 1$ to get weak convergence of transition probability in Theorem 2.

To complete the proof of main theorem, it remains to observe that convergence of finite dimensional distribution is satisfied. We can complete the proof of Theorem 2 as verifying the following corollary:

Corollary 7. For $0 \leq t_{1}<t_{2}<\cdots<t_{k}$

$$
\left(\frac{1}{\sqrt{n}} S_{\left[n t_{1}\right]}^{\left(\alpha_{\eta}\right)}, \ldots, \frac{1}{\sqrt{n}} S_{\left[n t_{k}\right]}^{\left(\alpha_{\eta}\right)}\right) \rightarrow\left(B_{t_{1}}^{\left(\alpha_{\eta}\right)}, \ldots, B_{t_{k}}^{\left(\alpha_{\eta}\right)}\right) \text { as } n \rightarrow \infty .
$$

Proof. Using Theorem 2 and the same proof methods (see, for instance, [21] (p. 23)) as in proving the convergence of finite dimensional distribution for classical random walks, the proof is completed. 


\section{Conclusions}

In this paper, we propose random walks on a spider which is the general model for skew random walks and we investigated some limiting distributions for random walks on a spider. In particular, we complete a functional central limit theorem for random walks on a spider and to define Brownian spider as the resulting weak limit. To complete the proof, we first investigated the tightness properties using the method based on a fourth order moment and then observed the convergence of finite dimensional distribution for random walks on a spider.

Funding: This research is supported by the Dong-A University research grant.

Conflicts of Interest: The author declares no conflict of interest.

\section{References}

1. Itô, K.; McKean, H.P. Brownian Motions on a half Line. Ill. J. Math. 1963, 7, 181-231. [CrossRef]

2. Itô, K.; McKean, H.P. Diffusion Processes and Their Sample Paths (Classics in Mathematics); Springer: Berlin/Heidelberg, Germany, 1974.

3. Walsh, J.B. A diffusion with discontinuous local time. Astérisque 1978, 52-53, 37-45.

4. Barlow, M.T.; Pitman J.W.; Yor, M. On walsh's brownian motion. In Lecture Notes in Mathematics; Séminaire de Probabilités; XXIII; Springer: Berlin/Heidelberg, Germany, 1989; Volume 1372, pp. 275-293.

5. Evans, S.N.; Sowers, R.B. Pinching and twisting Markov processes. Ann. Probab. 2003, 31, 486-527. [CrossRef]

6. Barlow, M.T.; Pitman J.W.; Yor, M. Une extension multidimensionelle de la loi de l'arc sinus. In Lecture Notes in Mathematics; Séminaire de Probabilités; XXIII; Springer: Berlin/Heidelberg, Germany, 1989; Volume 1372, pp. 294-314.

7. Papanicolaou, V.G.; Papageorgiou, E.G.; Lepipas, D.C. Random motion on simple graph. Methodol. Comput. Appl. Probab. 2012, 14, 285-297. [CrossRef]

8. Vakeroudis, S.; Yor, M. A scaling proof for Walsh's Brownian motion extended arc-sine law. Electron. Commun. Probab. 2012, 17, 9. [CrossRef]

9. Hajri, H. Discrete approximations to solution flows of Tanaka's SDE related to Walsh Brownian motion. In Lecture Notes in Mathematics; Séminaire de Probabilités; XLIV; Springer: Berlin/Heidelberg, Germany, 2012; Volume 2046, pp. 167-190.

10. Matzavinos, A.; Roitershtein, A.; Seol, Y. Random walks in a sparse random environment. Electron. J. Probab. 2016, 21 , 1-20. [CrossRef]

11. Ramirez, J.M. Multi-skewed Brownian motion and diffusion in layered media. Proc. Am. Math. Soc. 2011, 139, 3739-3752. [CrossRef]

12. Csáki, E.; Csörgó, M.; Földes, A.; Révész, P. Some limit theorems for heights of random walks on a spider. J. Theor. Probab. 2016, 29, 1685-1709. [CrossRef]

13. Csáki, E.; Csörgő, M.; Földes, A.; Révész, P. Strong approximation of additive functionals. J. Theor. Probab. 1992, 5, 679-706. [CrossRef]

14. Harrison, M.; Shepp, L. On Skew Brownian Motion. Ann. Probab. 1981, 9, 309-313. [CrossRef]

15. Hoteit, H.; Mose, R.; Younes, A.; Lehmann, F.; Ackerer, P.H. Three-dimensional modeling of mass transfer in porous media using the mixed hybrid finite elements and random walk methods. Math. Geosci. 2002, $34,435-456$.

16. Lejay, A. On the construction of the skew Brownian motion. Probab. Surv. 2006, 3, 413-466. [CrossRef]

17. Saxton, R.A.; Jacobson, K. Single-Particle Tracking: Applications to membrane dynamics. Annu. Rev. Biophys. Biomol. Struct. 1997, 26, 373-399. [CrossRef] [PubMed]

18. Cherny, A.S.; Shiryaev, A.N.; YoR, M. Limit behavior of the "horizontal-vertical" random walk and some extensions of the Donker-Prokhorov invariance principle. Theory Probab. Appl. 2002, 47, 498-517.

19. Étoré, P. On random walk simulation of one-dimensional diffusion processes with discontinuous coefficients. Electron. J. Probab. 2006, 11, 249-275. [CrossRef]

20. Seol, Y. On tightness of skew random walks. J. Probab. Stat. 2012, 2012, 371025. [CrossRef] 
21. Bhattacharya, R.; Waymire, E. Stochastic Processes with Applications; John Wiley and Sons: Hoboken, NJ, USA, 1990; Volume 9.

22. Bhattacharya, R.; Waymire, E. A Basic Course in Probability Theory; Springer: New York, NY, USA, 2007.

Publisher's Note: MDPI stays neutral with regard to jurisdictional claims in published maps and institutional affiliations.

(C) 2020 by the author. Licensee MDPI, Basel, Switzerland. This article is an open access article distributed under the terms and conditions of the Creative Commons Attribution (CC BY) license (http://creativecommons.org/licenses/by/4.0/). 